Supplementary Information for:

\title{
Higher fine particle fraction in sediment increased phosphorus flux to estuary in restored Yellow River Basin
}

Yidi Wang ${ }^{1}$, Wei Ouyang ${ }^{1 *}$, Chunye Lin ${ }^{1}$, Weihong Zhu², Andrea Critto ${ }^{3}$, Mats Tysklind $^{4}$, Xuelei Wang ${ }^{5}$, Mengchang $\mathrm{He}^{1}$, Baodong Wang ${ }^{6}$, Haotian $\mathrm{Wu}^{1}$

${ }^{1}$ School of Environment, State Key Laboratory of Water Environment Simulation, Beijing Normal University, Beijing 100875, P.R. China

${ }^{2}$ School of Geography and Ocean Science, Yanbian University, Changbai Mountain Key Laboratory of Biological Resources and Functional Molecules, Yanji 133003, P.R. China

${ }^{3}$ Department of Environmental Sciences, Informatics and Statistics, University Ca' Foscari Venice, I-30170 Venice, Italy

${ }^{4}$ Department of Chemistry, Umeå University, SE-901 87 Umeå, Sweden

${ }^{5}$ Satellite Environment Center, Ministry of Ecology and Environment, Beijing 100094, P.R. China

${ }^{6}$ First Institute of Oceanography, Ministry of Natural Resources, 6 Xianxialing Road, Qingdao 266061, P.R. China

Number of pages: 14

Number of figures: 3

Number of tables: 8 


\section{Content}

- Redundancy analysis (RDA) method

- Multilevel factorial analysis (MFA) method

- Constant rate of supply (CRS) model

- Supplementary Figure 1. Sediment sampling information. a, Locations of YR basin and delta-front estuary. b, Natural environment of Yellow River mouth. c, YR mouth sediment core. d, Surface sediment sampling in estuary. e, Sediment core sampling in estuary. f, Natural environment of YR main stream. g, Sediment core in YR basin.

- Supplementary Figure 2. Sediment analysis methods and related equipment. a, A low-background High Purity Germanium (HPGe) $\gamma$ spectrometer (GCW3023, Canberra, USA) was used to determine ${ }^{210} \mathrm{~Pb},{ }^{226} \mathrm{Ra}$ and ${ }^{137} \mathrm{Cs}$ activities. $\mathrm{b}$, Ultra high temperature digester (DigiBlock EHD36, LabTech, USA) was used for sediment pretreatment. c, Inductively coupled plasma-atomic emission spectroscopy (ICP-AES) (ICP 7000, Thermo Scientific, USA) was used to measure elements in the extracts. d, TOC analyzer (TOC-L CPN, Shimadzu, Japan) was used to determine TOC. e, A laser particle size analyzer (S3500, Microtrac, USA) was used to measure particle size.

- Supplementary Figure 3. Correlation analysis between P, Fe and Mn in the B2 core $(n=22)$.

- Supplementary Table 1. Remote Sensing data parameter

- Supplementary Table 2. Sediment discharge and runoff in the Yellow River estuary

- $\quad$ Supplementary Table 3. The detail information of sampling site

- Supplementary Table 4. Sedimentary type of Y1 sediment core

- Supplementary Table 5. Sedimentary type and pollutants concentration of B2 sediment core

- Supplementary Table 6. The physicochemical properties of surface sediments from Yellow River Estuary

- Supplementary Table 7. Total, supported and excess ${ }^{210} \mathrm{~Pb}$ concentrations and ${ }^{137} \mathrm{Cs}$ concentration in the sediment core B2, and CRS modeled sedimentation rates

- Supplementary Table 8 . The physicochemical properties of surface sediments from Yellow River Basin 


\section{- Redundancy analysis (RDA) method}

RDA is a kind of constrained ordination analysis (also known as "direct gradient analysis"), in which the ordination axes become weighted sums of environmental variables achieved through multiple regression so that response variability can be displayed along environmental gradients. To determine whether to choose a linear or unimodal model, the analysis was first performed with detrended correspondence analysis (DCA). Because the DCA results showed that the maximum gradient of the four axes was less than three, RDA was selected for determining the relationship between $\mathrm{P}$ and the physicochemical properties of sediments.

\section{- Multilevel factorial analysis (MFA) method}

MFA is a powerful tool to efficiently facilitate the exploration of parameter interactions by disclosing the specific variations in each parameter's effect under the impact of other parameters. This technique is useful when there is a nonlinear relationship between the independent variables (i.e., factors) and the dependent variable (i.e., response). In this study, the MFA was examined with a two-level factorial experiment (low and high) to deal with the test matrix of four parameters (Fe, Mn, silt and TOC). The low levels are determined with the lower bounds of the $95 \% \mathrm{CI}$ of the parameters; the high levels correspond to the upper bounds of parameters' $95 \%$ CI. After investigating the parameters' interactive effects, the significant parameters and their interactions can be identified.

\section{- Constant rate of supply (CRS) model}

Dating models are commonly used to (i) obtain an age model, i.e., the section/layer age as a function of depth, and (ii) calculate accumulation rates and estimate sediment mixing rates. The inherent variability of accumulation rates in most natural sedimentary systems has resulted in the CRS model being the most widely used means of deriving ${ }^{210} \mathrm{~Pb}$ chronologies. The CRS model is based on the assumption that the supply of ${ }^{210} \mathrm{~Pb}$ to the sea surface is constant, while the sediment accumulation rate might vary. The application of this model requires accurate determination of both $A$ and $A_{m}$ (equation 2); therefore, the integrity of the collection of all material over the depth sampled is of fundamental importance. Loss of surface material during sediment sampling can generate inaccuracies in chronologies. Special attention to sample integrity was paid in our sampling procedure to ensure the accuracy of dating calculations. 


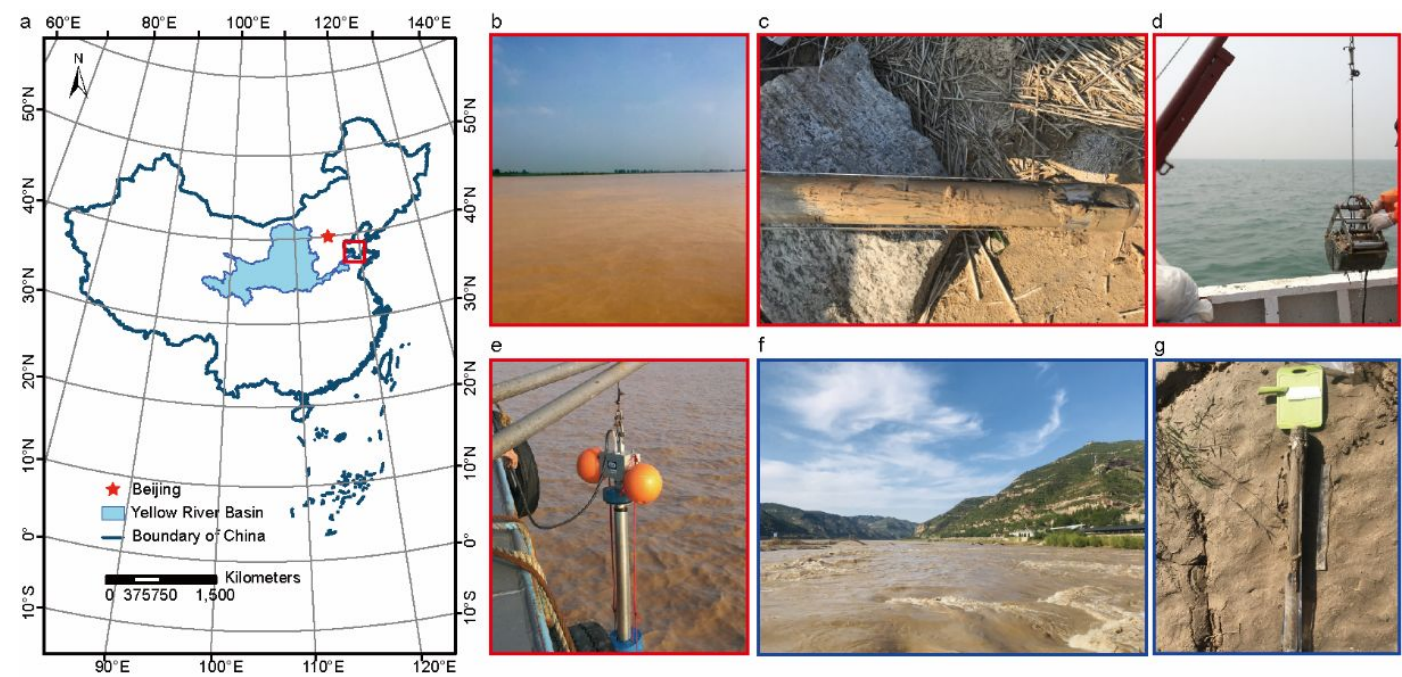

Supplementary Figure 1. Sediment sampling information. a, Locations of YR basin and delta-front estuary. b, Natural environment of Yellow River mouth. c, YR mouth sediment core. d, Surface sediment sampling in estuary. e, Sediment core sampling in estuary. f, Natural environment of YR main stream. g, Sediment core in YR basin. 
a

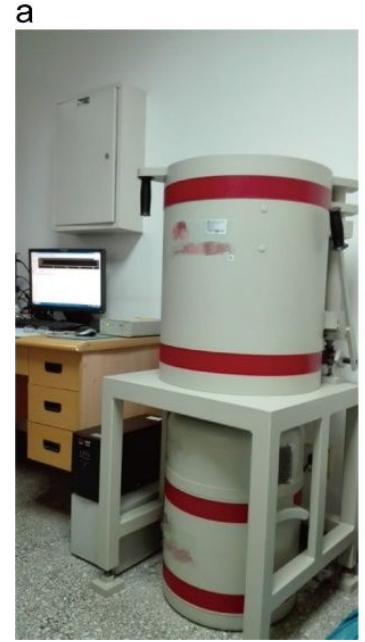

b
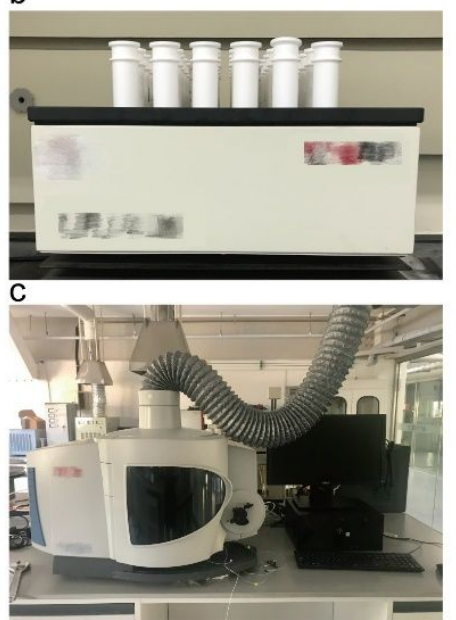

d
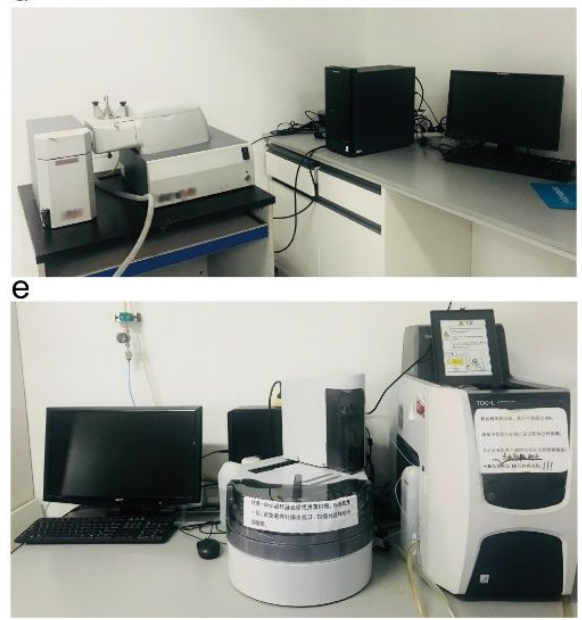

Supplementary Figure 2. Sediment analysis methods and related equipment. a, A lowbackground High Purity Germanium (HPGe) $\gamma$ spectrometer (GCW3023, Canberra, USA) was used to determine ${ }^{210} \mathrm{~Pb},{ }^{226} \mathrm{Ra}$ and ${ }^{137} \mathrm{Cs}$ activities. $\mathrm{b}$, Ultra high temperature digester (DigiBlock EHD36, LabTech, USA) was used for sediment pretreatment. c, Inductively coupled plasma-atomic emission spectroscopy (ICP-AES) (ICP 7000, Thermo Scientific, USA) was used to measure elements in the extracts. d, TOC analyzer (TOC-L CPN, Shimadzu, Japan) was used to determine TOC. e, A laser particle size analyzer (S3500, Microtrac, USA) was used to measure particle size. 


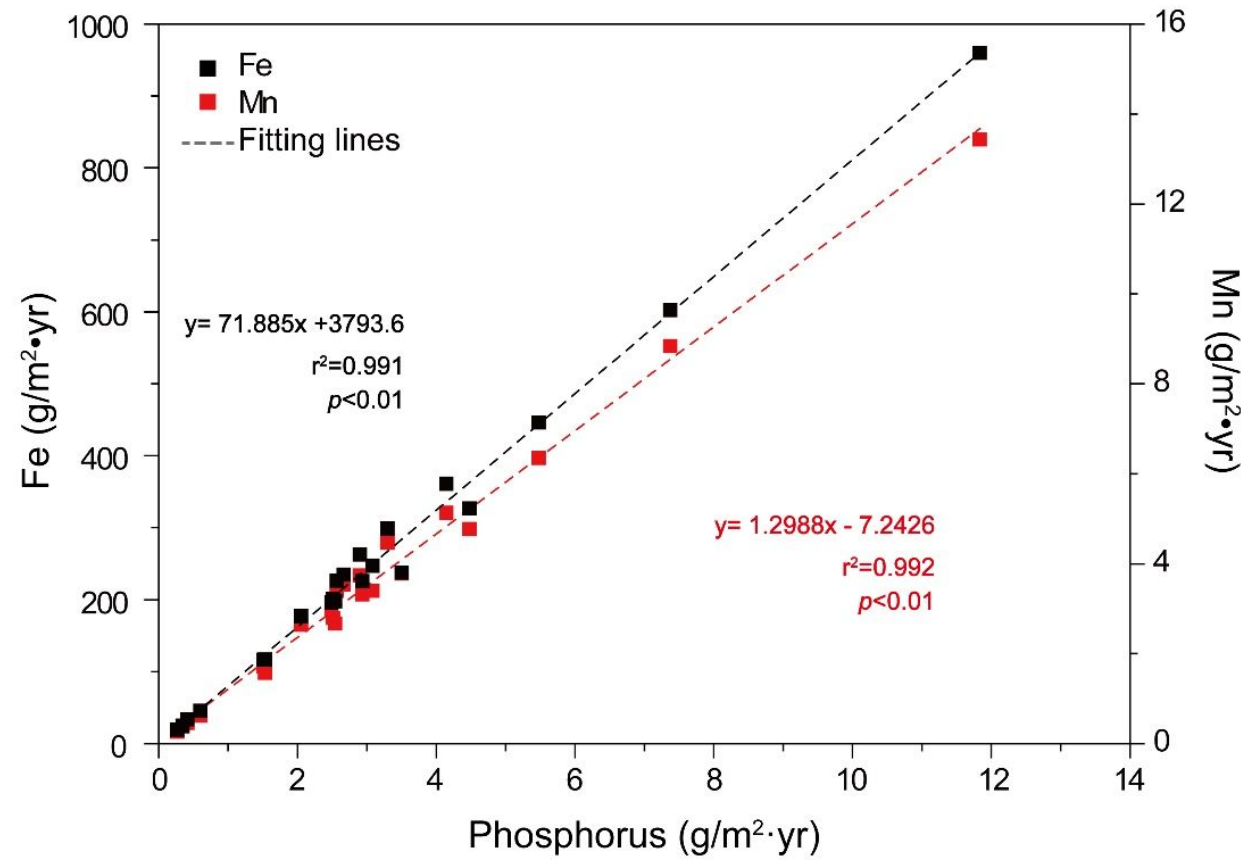

Supplementary Figure 3. Correlation analysis between P, Fe and Mn in the B2 core $(n=22)$. 
Supplementary Table 1. Remote Sensing data parameter

\begin{tabular}{ccccc}
\hline ID & Imaging Time & Serial Number & Image types & Spatial Resolution (m) \\
\hline 1 & $08 / 06 / 1987$ & LT51210341987159HAJ00 & TM & 30 \\
2 & $02 / 04 / 1992$ & LT51210341992093HAJ00 & TM & 30 \\
3 & $02 / 07 / 1996$ & LT51210341996184HAJ00 & TM & 30 \\
4 & $21 / 11 / 2001$ & LT51210342001325BJC00 & TM & 30 \\
5 & $08 / 05 / 2005$ & LT51210342005128BJC00 & TM & 30 \\
6 & $11 / 09 / 2010$ & LT51210342010254IKR00 & TM & 30 \\
7 & $26 / 08 / 2016$ & LC81210342016239LGN00 & TM & 30 \\
\hline
\end{tabular}


Supplementary Table 2. Sediment discharge and runoff in the Yellow River estuary

\begin{tabular}{ccc}
\hline Year & $\begin{array}{c}\text { Sediment discharge } \\
\left(\mathbf{G m}^{\mathbf{3}}\right)\end{array}$ & $\begin{array}{c}\text { Ruoff discharge } \\
(\mathbf{G t})\end{array}$ \\
\hline $1987-1992$ & 1064.2 & 26.96 \\
$1993-1996$ & 693.9 & 26.07 \\
$1997-2001$ & 470.59 & 11.6 \\
$2002-2005$ & 640.1 & 8.723 \\
$2006-2010$ & 867.2 & 5.962 \\
2011 & 184.2 & 0.926 \\
2012 & 282.5 & 1.83 \\
2013 & 236.9 & 1.73 \\
2014 & 114.3 & 0.301 \\
2015 & 133.6 & 0.314 \\
2016 & 81.88 & 0.106 \\
2017 & 89.58 & 0.077 \\
2018 & 333.8 & 2.97 \\
\hline
\end{tabular}


Supplementary Table 3. The detail information of sampling site

\begin{tabular}{|c|c|c|c|c|c|c|c|}
\hline $\begin{array}{l}\text { Site } \\
\text { ID }\end{array}$ & Date & $\begin{array}{l}\text { Latitude } \\
\left({ }^{\circ} \mathrm{N}\right)\end{array}$ & $\begin{array}{c}\text { Longitude } \\
\left({ }^{\circ} \mathbf{E}\right)\end{array}$ & $\begin{array}{l}\text { Site } \\
\text { ID }\end{array}$ & Date & $\begin{array}{c}\text { Latitude } \\
\left({ }^{\circ} \mathbf{N}\right)\end{array}$ & $\begin{array}{c}\text { Longitude } \\
\left({ }^{\circ} \mathbf{E}\right)\end{array}$ \\
\hline $\mathrm{B} 1$ & $21 / 07 / 2018$ & $37^{\circ} 72^{\prime} 69^{\prime \prime}$ & $119^{\circ} 57^{\prime} 07^{\prime \prime}$ & H1 & $19 / 09 / 2019$ & $36^{\circ} 05^{\prime} 89^{\prime \prime}$ & $101^{\circ} 45^{\prime} 03^{\prime \prime}$ \\
\hline $\mathrm{B} 2$ & $21 / 07 / 2018$ & $37^{\circ} 72^{\prime} 67^{\prime \prime}$ & $119^{\circ} 43^{\prime} 45^{\prime \prime}$ & $\mathrm{H} 2$ & 20/09/2019 & $36^{\circ} 07^{\prime} 79^{\prime \prime}$ & $103^{\circ} 77^{\prime} 99^{\prime \prime}$ \\
\hline B3 & $21 / 07 / 2018$ & $37^{\circ} 91^{\prime} 05^{\prime \prime}$ & $119^{\circ} 40^{\prime} 96^{\prime \prime}$ & H3 & $21 / 09 / 2019$ & $37^{\circ} 48^{\prime} 21^{\prime \prime}$ & $105^{\circ} 19^{\prime} 16^{\prime \prime}$ \\
\hline B4 & $21 / 07 / 2018$ & $38^{\circ} 13^{\prime} 55^{\prime \prime}$ & $119^{\circ} 23^{\prime} 24^{\prime \prime}$ & $\mathrm{H} 4$ & $26 / 09 / 2019$ & $35^{\circ} 37^{\prime} 48^{\prime \prime}$ & $110^{\circ} 50^{\prime} 76^{\prime \prime}$ \\
\hline B5 & $22 / 07 / 2018$ & $37^{\circ} 62^{\prime} 30^{\prime \prime}$ & $119^{\circ} 43^{\prime} 25^{\prime \prime}$ & H5 & $25 / 09 / 2019$ & $35^{\circ} 68^{\prime} 01^{\prime \prime}$ & $110^{\circ} 58^{\prime} 83^{\prime \prime}$ \\
\hline B6 & $21 / 07 / 2018$ & $37^{\circ} 62^{\prime} 30^{\prime \prime}$ & $119^{\circ} 57^{\prime} 07^{\prime \prime}$ & H6 & $25 / 09 / 2020$ & $36^{\circ} 35^{\prime} 21^{\prime \prime}$ & $110^{\circ} 46^{\prime} 62^{\prime \prime}$ \\
\hline B7 & $21 / 07 / 2018$ & $37^{\circ} 62^{\prime} 54^{\prime \prime}$ & $119^{\circ} 70^{\prime} 90^{\prime \prime}$ & H7 & $24 / 09 / 2019$ & $38^{\circ} 30^{\prime} 67^{\prime \prime}$ & $110^{\circ} 61^{\prime \prime} 37^{\prime \prime}$ \\
\hline B8 & $21 / 07 / 2018$ & $37^{\circ} 73^{\prime} 36^{\prime \prime}$ & $119^{\circ} 70^{\prime} 90^{\prime \prime}$ & H8 & $24 / 09 / 2019$ & $39^{\circ} 19^{\prime} 64^{\prime \prime}$ & $111^{\circ} 18^{\prime} 30^{\prime \prime}$ \\
\hline B9 & $22 / 07 / 2018$ & $37^{\circ} 84^{\prime} 05^{\prime \prime}$ & $119^{\circ} 16^{\prime} 62^{\prime \prime}$ & H9 & $23 / 09 / 2019$ & $40^{\circ} 20^{\prime} 85^{\prime \prime}$ & $111^{\circ} 18^{\prime} 48^{\prime \prime}$ \\
\hline B10 & $22 / 07 / 2018$ & $37^{\circ} 92^{\prime} 42^{\prime \prime}$ & $119^{\circ} 17^{\prime} 77^{\prime \prime}$ & $\mathrm{H} 10$ & $22 / 09 / 2019$ & $40^{\circ} 53^{\prime} 09^{\prime \prime}$ & $109^{\circ} 92^{\prime} 92^{\prime \prime}$ \\
\hline B11 & $22 / 07 / 2018$ & $38^{\circ} 04^{\prime} 61^{\prime \prime}$ & $119^{\circ} 20^{\prime} 62^{\prime \prime}$ & H11 & 03/10/2019 & $34^{\circ} 61^{\prime} 12^{\prime \prime}$ & $110^{\circ} 45^{\prime} 41^{\prime \prime}$ \\
\hline B12 & $21 / 07 / 2018$ & $37^{\circ} 62^{\prime} 10^{\prime \prime}$ & $119^{\circ} 32^{\prime} 30^{\prime \prime}$ & H12 & 03/10/2019 & $34^{\circ} 99^{\prime} 83^{\prime \prime}$ & $109^{\circ} 85^{\prime} 87^{\prime \prime}$ \\
\hline B13 & $22 / 07 / 2018$ & $37^{\circ} 98^{\prime} 25^{\prime \prime}$ & $119^{\circ} 33^{\prime} 97^{\prime \prime}$ & H13 & 02/10/2019 & $34^{\circ} 56^{\prime} 17^{\prime \prime}$ & $109^{\circ} 64^{\prime} 80^{\prime \prime}$ \\
\hline B14 & $22 / 07 / 2018$ & $38^{\circ} 04^{\prime} 89^{\prime \prime}$ & $119^{\circ} 37^{\prime} 88^{\prime \prime}$ & H14 & $02 / 10 / 2019$ & $34^{\circ} 61^{\prime} 10^{\prime \prime}$ & $108^{\circ} 63^{\prime} 32^{\prime \prime}$ \\
\hline B15 & $22 / 07 / 2018$ & $37^{\circ} 93^{\prime} 20^{\prime \prime}$ & $119^{\circ} 30^{\prime} 07^{\prime \prime}$ & H15 & $02 / 10 / 2019$ & $34^{\circ} 70^{\prime} 75^{\prime \prime}$ & $107^{\circ} 71^{\prime} 92^{\prime \prime}$ \\
\hline B16 & $28 / 07 / 2018$ & $37^{\circ} 86^{\prime} 90^{\prime \prime}$ & $119^{\circ} 26^{\prime} 33^{\prime \prime}$ & H16 & 07/10/2019 & $34^{\circ} 91^{\prime} 87^{\prime \prime}$ & $113^{\circ} 63^{\prime} 84^{\prime \prime}$ \\
\hline B17 & $28 / 07 / 2018$ & $37^{\circ} 98^{\prime} 73^{\prime \prime}$ & $119^{\circ} 46^{\prime} 50^{\prime \prime}$ & H17 & 08/10/2019 & $36^{\circ} 29^{\prime} 87^{\prime \prime}$ & $116^{\circ} 33^{\prime} 29^{\prime \prime}$ \\
\hline B18 & $23 / 07 / 2018$ & $37^{\circ} 73^{\prime} 01^{\prime \prime}$ & $119^{\circ} 32^{\prime} 32^{\prime \prime}$ & H18 & 09/10/2019 & $37^{\circ} 54^{\prime} 75^{\prime \prime}$ & $118^{\circ} 31^{\prime} 30^{\prime \prime}$ \\
\hline B19 & $23 / 07 / 2018$ & $37^{\circ} 82^{\prime} 63^{\prime \prime}$ & $119^{\circ} 47^{\prime} 47^{\prime \prime}$ & & & & \\
\hline B20 & $29 / 07 / 2018$ & $37^{\circ} 79^{\prime} 67^{\prime \prime}$ & $119^{\circ} 31^{\prime} 28^{\prime \prime}$ & & & & \\
\hline B21 & $29 / 07 / 2018$ & $37^{\circ} 85^{\prime} 07^{\prime \prime}$ & $119^{\circ} 36^{\prime} 73^{\prime \prime}$ & & & & \\
\hline Y1 & $04 / 08 / 2018$ & $37^{\circ} 79^{\prime} 46^{\prime \prime}$ & $119^{\circ} 00^{\prime} 40^{\prime \prime}$ & & & & \\
\hline
\end{tabular}


Supplementary Table 4. Sedimentary type of Y1 sediment core

\begin{tabular}{cccc}
\hline $\begin{array}{c}\text { Depth } \\
\text { (cm) }\end{array}$ & $\begin{array}{c}\text { Clay } \\
\text { \% }\end{array}$ & $\begin{array}{c}\text { Silt } \\
\text { \% }\end{array}$ & $\begin{array}{c}\text { Sand } \\
\text { \% }\end{array}$ \\
\hline $0-1$ & 0.00 & 69.50 & 30.50 \\
$1-2$ & 0.00 & 68.89 & 31.11 \\
$2-3$ & 0.00 & 72.82 & 27.18 \\
$3-4$ & 0.00 & 71.65 & 28.35 \\
$4-5$ & 0.04 & 76.89 & 23.07 \\
$5-6$ & 0.04 & 70.64 & 29.32 \\
$6-7$ & 0.02 & 56.36 & 43.62 \\
$7-8$ & 0.04 & 57.71 & 42.25 \\
$8-9$ & 0.02 & 52.47 & 47.51 \\
$9-10$ & 0.04 & 62.28 & 37.68 \\
$10-11$ & 0.05 & 65.00 & 34.95 \\
$11-12$ & 0.06 & 71.58 & 28.36 \\
$12-13$ & 0.05 & 70.00 & 29.95 \\
$13-14$ & 0.04 & 71.49 & 28.47 \\
$14-15$ & 0.04 & 74.47 & 25.49 \\
$15-16$ & 0.04 & 70.61 & 29.35 \\
$16-17$ & 0.04 & 72.57 & 27.39 \\
$17-18$ & 0.04 & 73.92 & 26.04 \\
$18-19$ & 0.04 & 73.37 & 26.59 \\
$19-20$ & 0.00 & 70.47 & 29.53 \\
$20-21$ & 0.04 & 68.01 & 31.95 \\
$21-22$ & 0.00 & 67.62 & 32.38 \\
$22-23$ & 0.00 & 64.76 & 35.24 \\
$23-24$ & 0.04 & 66.60 & 33.36 \\
$24-25$ & 0.00 & 68.36 & 31.64 \\
$25-26$ & 0.00 & 69.80 & 30.20 \\
$26-27$ & 0.00 & 69.31 & 30.69 \\
$27-28$ & 0.06 & 72.44 & 27.50 \\
$28-29$ & 0.06 & 72.26 & 27.68 \\
$29-30$ & 0.04 & 71.06 & 28.90 \\
$30-31$ & 0.00 & 70.46 & 29.54 \\
$31-32$ & 0.04 & 73.42 & 26.54 \\
$32-33$ & 0.05 & 76.49 & 23.46 \\
$33-34$ & 0.04 & 77.42 & 22.54 \\
$34-35$ & 0.04 & 75.90 & 24.06 \\
$35-36$ & 0.05 & 77.13 & 22.82 \\
\hline & & & \\
\hline
\end{tabular}


Supplementary Table 5. Sedimentary type and pollutants concentration of B2 sediment core

\begin{tabular}{cccccccc}
\hline $\begin{array}{c}\text { Depth } \\
(\mathbf{c m})\end{array}$ & $\begin{array}{c}\text { Clay } \\
\text { \% }\end{array}$ & $\begin{array}{c}\text { Silt } \\
\text { \% }\end{array}$ & $\begin{array}{c}\text { Sand } \\
\mathbf{\%}\end{array}$ & $\begin{array}{c}\mathbf{A s} \\
\mathbf{m g} / \mathbf{k g}\end{array}$ & $\begin{array}{c}\mathbf{C d} \\
\mathbf{m g} / \mathbf{k g}\end{array}$ & $\begin{array}{c}\mathbf{C r} \\
\mathbf{m g} / \mathbf{k g}\end{array}$ & $\begin{array}{c}\mathbf{T P} \\
\mathbf{m g} / \mathbf{k g}\end{array}$ \\
\hline $0-1$ & 1.84 & 97.04 & 1.12 & 17.32 & 0.24 & 74.45 & 629.59 \\
$1-2$ & 1.25 & 97.55 & 1.20 & 17.28 & 0.27 & 85.62 & 654.94 \\
$2-3$ & 1.15 & 98.16 & 0.69 & 18.38 & 0.27 & 81.06 & 640.68 \\
$3-4$ & 1.21 & 97.97 & 0.82 & 18.00 & 0.27 & 79.49 & 635.51 \\
$4-5$ & 1.23 & 98.06 & 0.71 & 17.15 & 0.26 & 80.63 & 629.35 \\
$5-6$ & 1.32 & 96.66 & 2.02 & 16.52 & 0.26 & 68.99 & 582.22 \\
$6-7$ & 1.22 & 94.74 & 4.04 & 16.02 & 0.25 & 67.45 & 583.66 \\
$7-8$ & 1.14 & 95.55 & 3.31 & 11.91 & 0.25 & 69.73 & 604.20 \\
$8-9$ & 1.20 & 97.27 & 1.54 & 15.40 & 0.25 & 68.16 & 648.99 \\
$9-11$ & 1.19 & 96.61 & 2.20 & 14.91 & 0.25 & 84.12 & 672.76 \\
$11-13$ & 1.28 & 97.15 & 1.57 & 16.51 & 0.26 & 78.78 & 654.56 \\
$13-15$ & 1.15 & 97.63 & 1.23 & 16.70 & 0.24 & 81.08 & 612.52 \\
$15-17$ & 1.35 & 96.03 & 2.62 & 15.71 & 0.27 & 79.49 & 660.88 \\
$17-19$ & 1.07 & 94.20 & 4.73 & 14.91 & 0.25 & 85.40 & 660.78 \\
$19-21$ & 0.98 & 93.95 & 5.07 & 14.70 & 0.25 & 78.76 & 674.46 \\
$21-23$ & 1.17 & 94.89 & 3.94 & 14.55 & 0.26 & 85.17 & 664.22 \\
$23-25$ & 1.07 & 97.68 & 1.25 & 16.32 & 0.27 & 79.51 & 636.84 \\
$25-27$ & 1.10 & 96.50 & 2.40 & 15.02 & 0.26 & 72.26 & 614.82 \\
$27-29$ & 1.19 & 97.38 & 1.43 & 15.39 & 0.27 & 90.67 & 653.10 \\
$29-31$ & 2.04 & 96.08 & 1.89 & 14.38 & 0.27 & 90.52 & 654.06 \\
$31-33$ & 2.13 & 96.24 & 1.63 & 14.04 & 0.28 & 89.41 & 655.30 \\
$33-35$ & 1.56 & 96.48 & 1.96 & 13.57 & 0.27 & 88.00 & 639.37 \\
$35-37$ & 1.24 & 97.68 & 1.08 & 14.47 & 0.28 & 85.12 & 662.35 \\
$37-39$ & 1.28 & 97.78 & 0.94 & 14.90 & 0.27 & 88.41 & 675.92 \\
\hline & & & & & & &
\end{tabular}


Supplementary Table 6. The physicochemical properties of surface sediments from Yellow River Estuary

\begin{tabular}{ccccccc}
\hline $\begin{array}{c}\text { Site } \\
\text { ID }\end{array}$ & $\mathbf{p H}$ & $\begin{array}{c}\mathbf{M z} \\
\boldsymbol{\mu m}\end{array}$ & $\begin{array}{c}\text { Clay } \\
\mathbf{\%}\end{array}$ & $\begin{array}{c}\text { Silt } \\
\mathbf{\%}\end{array}$ & $\begin{array}{c}\text { Sand } \\
\mathbf{\%}\end{array}$ & $\begin{array}{c}\text { TOC } \\
\mathbf{\%}\end{array}$ \\
\hline B1 & 8.8 & 9.58 & 2.07 & 94.57 & 3.37 & 6.47 \\
B2 & 8.96 & 34.04 & 2.29 & 61.97 & 35.74 & 4.89 \\
B3 & 9.01 & 13.29 & 7.25 & 89.07 & 3.68 & 4.49 \\
B4 & 8.71 & 23.52 & 7.01 & 74.91 & 18.09 & 4.33 \\
B5 & 9.04 & 45.65 & 0.12 & 56.03 & 43.85 & 2.77 \\
B6 & 8.72 & 18.88 & 0.35 & 80.20 & 19.46 & 5.80 \\
B7 & 8.70 & 29.81 & 5.34 & 73.55 & 21.12 & 3.91 \\
B8 & 8.99 & 41.11 & 1.22 & 62.14 & 37.87 & 3.92 \\
B9 & 8.90 & 8.98 & 3.80 & 94.02 & 2.18 & 6.66 \\
B10 & 9.10 & 18.62 & 14.14 & 78.65 & 7.21 & 3.49 \\
B11 & 9.11 & 8.79 & 1.06 & 98.94 & 0.00 & 4.02 \\
B12 & 8.86 & 36.80 & 1.99 & 71.44 & 26.57 & 3.88 \\
B13 & 9.03 & 59.40 & 2.01 & 40.95 & 57.04 & 2.41 \\
B14 & 9.04 & 17.31 & 1.74 & 94.93 & 3.34 & 4.49 \\
B15 & 8.98 & 42.76 & 2.52 & 62.14 & 35.34 & 3.64 \\
B16 & 8.81 & 36.68 & 3.05 & 64.07 & 32.89 & 2.60 \\
B17 & 8.76 & 43.04 & 7.94 & 57.08 & 34.99 & 3.72 \\
B18 & 9.05 & 60.13 & 0.12 & 38.79 & 61.09 & 2.06 \\
B19 & 8.90 & 20.13 & 1.90 & 82.25 & 15.85 & 5.47 \\
B20 & 8.92 & 43.87 & 2.38 & 55.26 & 42.36 & 3.27 \\
B21 & 8.46 & 58.73 & 2.37 & 35.82 & 61.80 & 3.07 \\
\hline & & & & & &
\end{tabular}


Supplementary Table 7. Total, supported and excess ${ }^{210} \mathrm{~Pb}$ concentrations and ${ }^{137} \mathrm{Cs}$ concentration in the sediment core B2, and CRS modeled sedimentation rates

\begin{tabular}{|c|c|c|c|c|c|c|}
\hline $\begin{array}{l}\text { Depth } \\
(\mathrm{cm})\end{array}$ & $\begin{array}{l}{ }^{210} \mathrm{~Pb}_{\text {tot }} \\
(\mathrm{Bq} / \mathrm{Kg})\end{array}$ & $\begin{array}{c}{ }^{226} \mathrm{Ra} \\
(\mathrm{Bq} / \mathrm{Kg})\end{array}$ & $\begin{array}{l}{ }^{210} \mathrm{~Pb}_{\mathrm{ex}} \\
(\mathrm{Bq} / \mathrm{Kg})\end{array}$ & $\begin{array}{c}{ }^{137} \mathrm{Cs} \\
(\mathrm{Bq} / \mathrm{Kg})\end{array}$ & $\begin{array}{c}\text { Mass } \\
\text { depth } \\
\left(\mathrm{g} / \mathrm{cm}^{2}\right)\end{array}$ & Year \\
\hline $0-1$ & 39.73 & 30.32 & 9.41 & 0.76 & 0.28 & 2018 \\
\hline $1-2$ & 38.75 & 29.92 & 8.83 & 2.07 & 1.07 & \\
\hline $2-3$ & 40.64 & 30.38 & 10.26 & 0.66 & 2.21 & \\
\hline $3-4$ & 40.93 & 31.52 & 9.41 & 1.12 & 3.50 & 2017 \\
\hline $4-5$ & 34.31 & 31.17 & 3.14 & 1.19 & 4.71 & \\
\hline $5-6$ & 33.73 & 29.90 & 3.83 & 2.30 & 5.77 & \\
\hline $6-7$ & 39.70 & 29.90 & 9.8 & 0.85 & 6.79 & \\
\hline $7-8$ & 40.41 & 31.37 & 9.04 & 2.50 & 7.76 & \\
\hline $8-9$ & 37.13 & 31.97 & 5.16 & 1.91 & 8.79 & \\
\hline $9-11$ & 33.19 & 31.06 & 2.13 & 0.00 & 10.07 & 2016 \\
\hline $11-13$ & 31.75 & 30.37 & 1.38 & 1.62 & 11.52 & \\
\hline $13-15$ & 40.07 & 29.98 & 10.09 & 1.24 & 13.00 & \\
\hline $15-17$ & 38.53 & 29.40 & 9.13 & 1.90 & 14.71 & \\
\hline $17-19$ & 31.57 & 29.80 & 1.77 & 1.84 & 16.35 & 2015 \\
\hline $19-21$ & 34.49 & 30.46 & 4.03 & 0.94 & 17.88 & \\
\hline $21-23$ & 35.12 & 30.10 & 5.02 & 1.48 & 19.57 & \\
\hline $23-25$ & 32.87 & 29.73 & 3.14 & 2.34 & 21.18 & 2014 \\
\hline $25-27$ & 37.15 & 29.46 & 7.69 & 1.76 & 22.72 & \\
\hline $27-29$ & 39.64 & 29.33 & 10.31 & 1.87 & 24.26 & 2013 \\
\hline $29-31$ & 35.71 & 29.58 & 6.13 & 1.97 & 25.79 & 2012 \\
\hline $31-33$ & 35.71 & 30.05 & 5.66 & 2.17 & 27.32 & 2011 \\
\hline $33-35$ & 33.15 & 30.12 & 3.03 & 1.33 & 28.90 & 2009 \\
\hline $35-37$ & 32.15 & 30.33 & 1.82 & 1.25 & 30.49 & - \\
\hline $37-39$ & 32.63 & 30.77 & 1.86 & 0.75 & 32.14 & - \\
\hline
\end{tabular}


Supplementary Table 8 . The physicochemical properties of surface sediments from Yellow River Basin

\begin{tabular}{|c|c|c|c|c|c|c|c|c|}
\hline $\begin{array}{c}\text { Site } \\
\text { ID }\end{array}$ & $\begin{array}{l}\mathrm{Mz} \\
\mu \mathrm{m}\end{array}$ & $\begin{array}{c}\text { Clay } \\
\% \\
\end{array}$ & $\begin{array}{c}\text { Silt } \\
\% \\
\end{array}$ & $\begin{array}{c}\text { Sand } \\
\%\end{array}$ & $\begin{array}{c}\text { TOC } \\
\%\end{array}$ & $\begin{array}{c}\mathrm{Fe} \\
\mathrm{mg} / \mathrm{kg}\end{array}$ & $\begin{array}{c}\mathrm{Mn} \\
\mathrm{mg} / \mathrm{kg}\end{array}$ & $\begin{array}{c}P \\
\mathrm{mg} / \mathrm{kg}\end{array}$ \\
\hline H1 & 9.58 & 0.866 & 17.076 & 82.058 & 0.86 & 23813 & 415.18 & 684.54 \\
\hline $\mathrm{H} 2$ & 34.04 & 6.61 & 43.372 & 50.018 & 1.5 & 27380 & 476.43 & 673.74 \\
\hline H3 & 13.29 & 3.408 & 66.654 & 29.938 & 1.5 & 24748 & 386.85 & 823 \\
\hline $\mathrm{H} 4$ & 23.52 & 4.433 & 55.331 & 40.236 & 1.19 & 23228 & 357.57 & 556.52 \\
\hline H5 & 45.65 & 2.69 & 96.826 & 0.484 & 1.55 & 26750 & 429.62 & 714.81 \\
\hline H6 & 18.88 & 3.021 & 18.115 & 78.864 & 0.93 & 20527 & 309.28 & 456.71 \\
\hline H7 & 29.81 & 1.492 & 66.204 & 32.304 & 1.25 & 26164 & 396.54 & 630.65 \\
\hline H8 & 41.11 & 3.409 & 66.724 & 29.867 & 1.14 & 23859 & 365.18 & 649.65 \\
\hline H9 & 8.98 & 1.012 & 22.812 & 76.176 & 0.92 & 20496 & 310.3 & 454.95 \\
\hline $\mathrm{H} 10$ & 18.62 & 4.302 & 75.777 & 19.921 & 1.35 & 27553 & 419.58 & 651.4 \\
\hline H11 & 8.79 & 0 & 36.507 & 63.493 & 1.12 & 23425 & 362.01 & 574.01 \\
\hline H12 & 36.80 & 3.007 & 60.556 & 36.437 & 1.11 & 24964 & 442.52 & 755.71 \\
\hline H13 & 59.40 & 6.793 & 66.251 & 26.956 & 1.25 & 24608 & 376.38 & 605.32 \\
\hline H14 & 17.31 & 1.044 & 86.702 & 12.254 & 1.61 & 29366 & 471.88 & 689.45 \\
\hline H15 & 42.76 & 1.454 & 72.712 & 25.834 & 1.05 & 31907 & 484.83 & 1048.5 \\
\hline H16 & 36.68 & 6.436 & 92.475 & 1.089 & 1.07 & 25095 & 366.17 & 668.43 \\
\hline H17 & 43.04 & 1.057 & 33.829 & 65.114 & 0.9 & 22398 & 322.4 & 513.55 \\
\hline H18 & 60.13 & 2.661 & 32.466 & 64.873 & 1 & 23400 & 328.78 & 561.67 \\
\hline
\end{tabular}

